\title{
EFICIÊNCIA ENERGÉTICA APLICADA EM UMA RESIDÊNCIA NA CIDADE DE TERESINA- PI: UM RELATO DE EXPERIÊNCIA ${ }^{1}$
}

\author{
Lucas Rocha de Abrantes Duarte ${ }^{2}$ - UNIFSA \\ Marcielly dos Reis Macêdo ${ }^{3}$ - UNIFSA \\ Shélyda Raiane Rodrigues Machado ${ }^{4}$ - UNIFSA \\ Gabriel Messias Mamede Costa ${ }^{5}$ - UNIFSA \\ Brenda Virna Sousa Rocha ${ }^{6}$ - UNIFSA \\ Íclerson Antônio Evangelista Sousa Lima ${ }^{7}$ - UNIFSA \\ Prof. Me. Ítalo Rodrigo Monte Soares ${ }^{8}-$ UNIFSA
}

\begin{abstract}
RESUMO
Atualmente a sociedade tende a se preocupar mais intensamente com o meio ambiente e com métodos para amenizar os danos causados a ele. Diante disso, o presente trabalho consiste na elaboração de um projeto de eficiência energética de uma residência na cidade de Teresina-PI. Previamente foi escolhida a residência a ser implementado o projeto, a partir de um estudo do consumo de 5 unidades consumidoras, sendo escolhida aquela que apresentou maior consumo nos últimos meses. Posteriormente foi realizado um diagnóstico da residência, a partir de levantamento de cargas, de análise da planta atual, de aspectos climáticos, burocráticos e particularidades da unidade consumidora. Em seguida, foram levantadas ações de melhorias para implantar na residência e a elaboração de uma nova planta da residência, com modificações sugestivas para melhor aproveitamento energético. Foi calculada ainda a viabilidade econômica do projeto de eficiência energética e verificado a viabilidade técnica dele. Foram sugeridas mudanças como a troca de alguns aparelhos, a implantação de alguns materiais e a mudança de hábitos dos membros da casa, tornandoos colaboradores do projeto de eficiência.
\end{abstract}

Palavras-Chave: Eficiência Energética. Uso Racional de Energia. Análise Energética.

\section{ABSTRACT}

Today, society tends to be more concerned about the environment and methods of mitigating the damage caused by it. Given this, the present work consists in elaborating a project of energy saving of a residence in the city of Teresina-PI. Previously the residence was chosen to be implemented or project, based on a study of consumption of 5 consumer units, being chosen the one that presented the highest consumption in recent months. Subsequently, a diagnosis of the residence was made, based on the lifting of loads, analysis of the current plant, climatic aspects, bureaucratic aspects and characteristics of the consumer unit. Then, improvement actions were implemented for the implanted residence and a new residence plan, with suggested changes for better energy use. The economic viability of the economic efficiency project was also calculated and its technical viability detected. Changes were suggested such as changing some appliances,

\footnotetext{
${ }^{1}$ Trabalho apresentado no Congresso Brasileiro Ciência e Sociedade (CBCS 2019), promovido pelo Centro Universitário Santo Agostinho, de 03 a 05 de outubro de 2019, em Teresina-PI.

${ }^{2}$ Acadêmico do $10^{\circ}$ período do curso de Bacharelado em Engenharia Elétrica - UNIFSA, lucasrocha4034@gmail.com.

${ }^{3}$ Acadêmica do $10^{\circ}$ período do curso de Bacharelado em Engenharia Elétrica - UNIFSA, marciellymary@hotmail.com.

${ }^{4}$ Acadêmica do $10^{\circ}$ período do curso de Bacharelado em Engenharia Elétrica - UNIFSA, shelydarodrigues@gmail.com.

${ }^{5}$ Acadêmico do $10^{\circ}$ período do curso de Bacharelado em Engenharia Elétrica - UNIFSA, messiasgabriel@hotmail.com.

${ }^{6}$ Acadêmica do $10^{\circ}$ período do curso de Bacharelado em Engenharia Elétrica - UNIFSA, brendavirna@hotmail.com.

${ }^{7}$ Acadêmico do $10^{\circ}$ período do curso de Bacharelado em Engenharia Elétrica - UNIFSA, iclersonb2k@hotmail.com.

${ }^{8}$ Docente do curso de Bacharelado em Engenharia Elétrica - UNIFSA, italo.soares@unifsa.com.br. ISBN: 978-65-80968-11-4
} DOI: $10.17648 /$ cbcs-2019-110675 
ANAIS CBCS 2019 | 3 a 5 de outubro de 2019 | Centro Universitário Santo Agostinho - Teresina - P|

implementing some materials and changing habits of house members, creating collaborators of the efficiency project.

Keywords: Energy Efficiency. Rational Use of Energy. Energy Analysis.

\section{INTRODUÇÃO}

Segundo a Agência Nacional de Energia - Aneel (2008), consumo de energia é um dos principais produtos indicadores do desenvolvimento econômico e do nível de qualidade de vida de qualquer sociedade. Ele reflete tanto o ritmo de atividade dos setores industrial, comercial e de serviços, quanto à capacidade da população para adquirir bens e serviços tecnologicamente mais avançados, como automóveis, eletrodomésticos e eletroeletrônicos.

O consumo de energia por parte da população possui vários níveis de intensidade, que variam de acordo com aspectos geográficos (tais como clima, localização), aspectos socioeconômicos (Demográficos, PIB per capita, PIB Setorial) e os indicadores ditos tecnológicos, tal como consumo das novas tecnologias. Desse modo, as pessoas escolhem os bens e serviços que satisfazem suas necessidades e que estejam em conformidade com suas condições.

Com o intuito de se tornarem mais competitivas, as empresas buscam na eficiência energética uma oportunidade para reduzir custos. Ora adotando um discurso ambientalista, ora prevendo dificuldades na disponibilidade energética, o uso consciente da energia elétrica entra em pauta na gestão de uma empresa e buscam-se conceitos da engenharia, da economia e da administração, aplicando-os aos sistemas energéticos.

Conforme o INEE (Instituto Nacional de Eficiência Energética), a energia deve ser manipulada conscientemente. Os aparelhos que funcionam a partir da eletricidade possuem funções específicas, as quais não devem ser exploradas em vão. Uma lâmpada acesa em uma sala vazia é um exemplo de utilização desnecessária de energia, assim como equipamentos em funcionamento quando ninguém está fazendo uso deles.

Algumas áreas das quais se pode promover a eficiência energética são: iluminação, refrigeração, fator de potência, sistemas de ar comprimido, equipamentos elétricos, motores elétricos e conscientização do uso da energia. Dessas áreas, aquelas que apresentam maior 


\section{cONQ⿻口卄:ESOC CIENCIASSOCIEDADE

ANAIS CBCS 2019 | 3 a 5 de outubro de 2019 | Centro Universitário Santo Agostinho - Teresina - PI

influência, em geral, é a iluminação e a refrigeração, principalmente em ambientes que não utilizam motores elétricos.

Conforme Marque (2007), o governo brasileiro propõe diretrizes através do Ministério de Minas e Energia, do Ministério da Educação, da Eletrobrás e outras instituições, com o objetivo de prover a indústria e os diferentes setores do país com informações e técnicas. Entre várias iniciativas estão o Programa Brasileiro de Etiquetagem (PBE), sob coordenação do Instituto Nacional de Metrologia, Normalização e Qualidade Industrial (INMETRO), o Programa Nacional de Conservação de Energia Elétrica (PROCEL), sob coordenação da Eletrobrás, e o Programa Nacional de Racionalização do Uso dos Derivados do Petróleo e do Gás Natural (CONPET), sob coordenação da Petrobras.

Diante do cenário apresentado e a necessidade de integração dos alunos da engenharia com a sociedade, vê-se o interesse de promover experiencias práticas para os futuros profissionais. 0 presente trabalho tem o objetivo de descrever a experiencia a cerca de uma aplicação de eficiência energética em uma residência na cidade de Teresina-PI, diagnosticando e propondo mudanças no perfil de consumo.

\section{METODOLOGIA}

O trabalho de eficiência se inicia com a avaliação da fatura do mês de março dos quatro integrantes do grupo, considerando o histórico, totalizando uma avaliação de 11 meses, onde foi realizado o estudo aprofundado da fatura de maior média de consumo mensal.

Figura 1. Fatura da residência escolhida 


\section{CONWEESSOO CIENCIAESOCIEDADE

ANAIS CBCS 2019 | 3 a 5 de outubro de 2019 | Centro Universitário Santo Agostinho - Teresina - P|

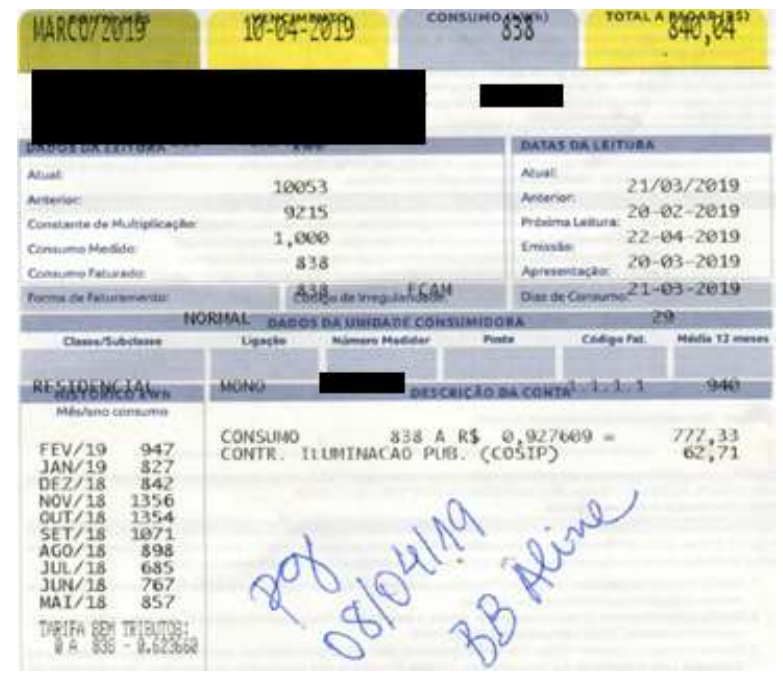

Fonte: Próprio autor

De acordo com a Figura 1, são mostrados os consumos referentes aos 10 meses anteriores à fatura de março. Com a realização do cálculo da média de consumo do mês de maio de 2018 até março de 2019, obteve-se o valor de 949,3 kWh. Foi escolhido essa residência para o desenvolvimento do trabalho relatado.

A residência escolhida é um apartamento em um condomínio fechado que está localizado no bairro Macaúba da cidade de Teresina, Piaui. A proprietária do apartamento trabalha como autônoma na produção de "din-din" em grande escala, o que demanda freezers para congelamento, armazenamento e venda do produto em seu perfeito estado de consistência.

Outro fato que foi possível perceber é que por ser apartamento possui regras rígidas acerca do quanto se pode interferir na atual situação da residência. Portanto, algumas das propostas que serão realizadas não terão a possibilidade de ser aplicada, uma vez que a rigidez de algumas regras (como, por exemplo, a que proíbe a troca de janelas ou construção/destruição de alguma parede já construída) dificulta esse processo. Após a inspeção na residência escolhida, foi realizada a confecção da planta da casa como esta é atualmente, antes das sugestões de melhorias, como mostra a Figura 2:

Figura 2. Planta atual da residência 


\section{CONQGEESSOCIENCIAESOCIEDADE

ANAIS CBCS 2019 | 3 a 5 de outubro de 2019 | Centro Universitário Santo Agostinho - Teresina - PI

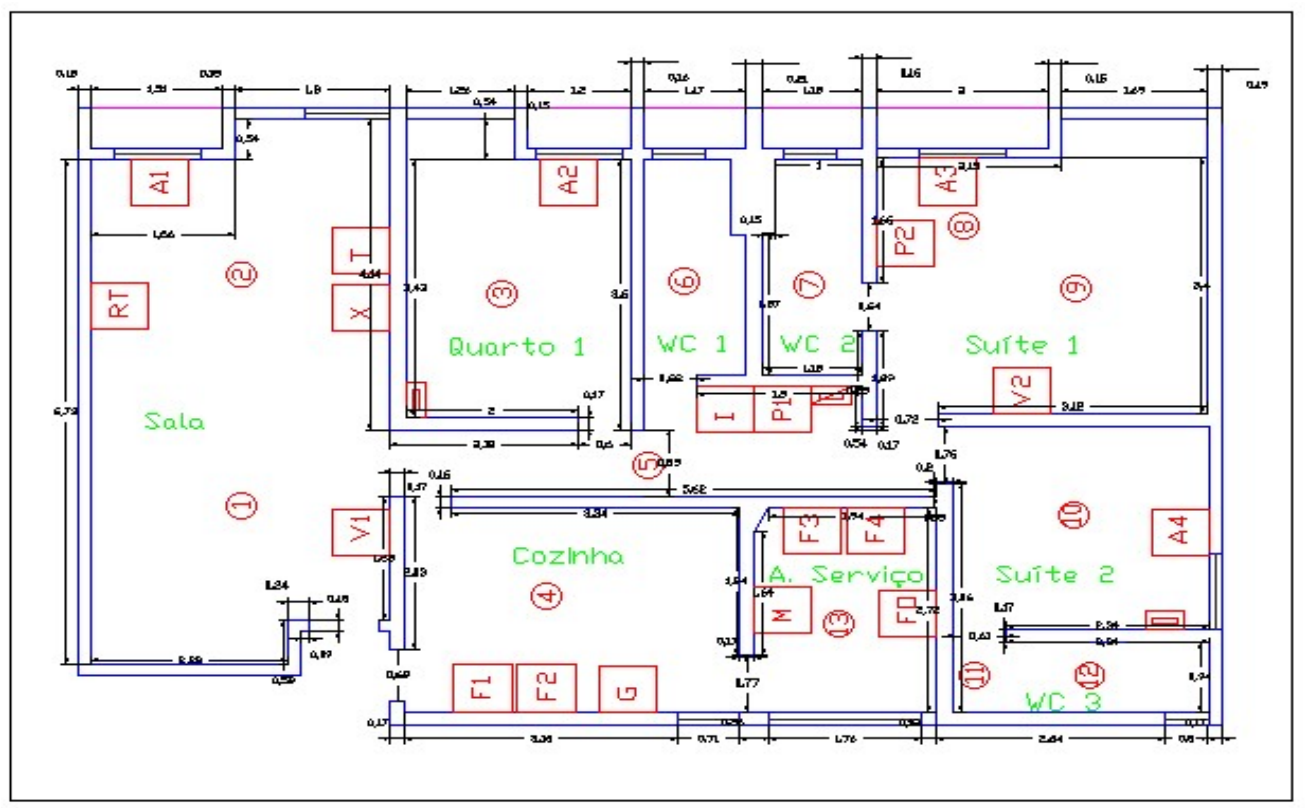

Fonte: Próprio autor

Analisando a residência, a planta e o levantamento de carga foi possível constatar também que há um ar condicionado em desuso que possui selo PROCEL C, o roteador da internet apresenta um consumo relativamente alto $(21,6 \mathrm{KWh} / \mathrm{mês})$ e algumas lâmpadas ainda são incandescente/fluorescente, necessitando, portanto, de sugestões de medidas para modificar situações como estas.

Quadro 1. Legenda da planta atual

\begin{tabular}{|c|c|c|c|}
\hline \multicolumn{5}{|c|}{ LEGENDA } \\
\hline RÓTULO & TIPO & RÓTULO & TIPO \\
\hline A1 & AR CONDICIONADO & O13 & ILUMINAÇÃO \\
\hline A2 & AR CONDICIONADO & RT & ROTEADOR \\
\hline A3 & AR CONDICIONADO & TV & TELEVISAO \\
\hline A4 & AR CONDICIONADO & $\mathrm{X}$ & VIDEOGAME \\
\hline O1 & ILUMINAÇÃO & V1 & VENTILADOR \\
\hline O2 & ILUMINAÇÃO & V2 & CLIMATIZADOR \\
\hline O3 & ILUMINAÇÃO & $\square$ & ILUMINAÇÃO \\
\hline O4 & ILUMINAÇÃO & $\square$ & ILUMINAÇÃO \\
\hline O5 & ILUMINAÇÃO & \multicolumn{2}{c}{} \\
\hline
\end{tabular}




\section{CONGEESSO CIENCIAESOCIEDADE \\ Inovação, Diversillatle e Sustentabilitidade}

ANAIS CBCS 2019 | 3 a 5 de outubro de 2019 | Centro Universitário Santo Agostinho - Teresina - P|

\begin{tabular}{|c|c|c|c|}
\hline O6 & ILUMINAÇÃO & $\mathrm{FO}$ & FORNO \\
\hline $\mathrm{O} 7$ & ILUMINAÇÃO & F1 & FREEZER \\
\hline $\mathrm{O} 8$ & ILUMINAÇÃO & $\mathrm{F} 2$ & FREEZER \\
\hline O9 & ILUMINAÇÃO & F3 & FREEZER \\
\hline $\mathrm{O} 10$ & ILUMINAÇÃO & $\mathrm{F} 4$ & FREEZER \\
\hline $\mathrm{O} 11$ & ILUMINAÇÃO & G & GELADEIRA \\
\hline $\mathrm{O} 12$ & ILUMINAÇÃO & M & MÁQUINA DE LAVAR ROUPAS \\
\hline
\end{tabular}

Fonte: Próprio autor

Com o auxílio da proprietária pode-se perceber que o freezer F1 (horizontal) apresenta capacidade de permanecer em temperatura suficiente para a sua função mesmo quando é desligado durante a noite (permanecendo necessariamente fechado).

Ainda a partir da pesquisa com os membros da residência, foi constatado que alguns aparelhos permanecem na tomada durante o período em que não estão de fato ligados e sendo usados, permanecendo em stand-by.

Portanto, será imprescindível projetar medidas que trabalhem exatamente em cima das constatações realizadas.

Foi realizado o levantamento de todos os equipamentos eletroeletrônicos contidos na residência, levando em consideração a classificação pelo selo PROCEL, a descrição do equipamento, a potência nominal e o tempo de uso de cada equipamento.

Tabela 1. Levantamento de cargas

\begin{tabular}{c|c|c|c|c|c}
\hline & POT & $\begin{array}{c}\text { HORAS } \\
\text { DE USO } \\
\text { DIA }\end{array}$ & $\begin{array}{c}\text { PREVISÃO } \\
\text { DE } \\
\text { CONSUMO } \\
\text { MÊS(KWh) }\end{array}$ & DESCRIÇÃO & PROCEL \\
\hline AR COND & 39,1 & 0 & 0 & $\begin{array}{c}\text { 18000BTU CONV } \\
\text { YORK }\end{array}$ & $\mathrm{C}$ \\
\hline AR COND & 17,1 & 7,5 & 128,25 & 9000BTU INVERTER & $\mathrm{AG}$ \\
\hline AR COND & 22,5 & 7,5 & 168,75 & 12000BTU CONV & ELBRUS \\
\hline AR COND & 17 & 7,5 & 127,5 & $\begin{array}{c}\text { 9000BTU INVERTER } \\
\text { ELBRUS }\end{array}$ & $\mathrm{A}$ \\
\hline
\end{tabular}




\section{conghisSO CIENCIAESOCIEDADE \\ Inovação, Diversiliaile e Sustentalililitaile}

ANAIS CBCS 2019 | 3 a 5 de outubro de 2019 | Centro Universitário Santo Agostinho - Teresina - P|

\begin{tabular}{|c|c|c|c|c|c|}
\hline ILUMINAÇÃO & 16 & 6 & 2,88 & 2X8W LED & A \\
\hline ILUMINAÇÃO & 16 & 6 & 2,88 & 2X8W LED & $\mathrm{A}$ \\
\hline ILUMINAÇÃO & 9 & 6 & 1,62 & 1X9W LED & $A$ \\
\hline ILUMINAÇÃO & 6,5 & 6 & 1,17 & 1X6,5W LED & A \\
\hline ILUMINAÇÃO & 20 & 6 & 3,6 & 1X20W FLUO & \\
\hline ILUMINAÇÃO & 6,5 & 6 & 1,17 & 1X6,5W LED & A \\
\hline ILUMINAÇÃO & 6,5 & 6 & 1,17 & 1X6,5W LED & A \\
\hline ILUMINAÇÃO & 9 & 6 & 1,62 & 1X9W LED & A \\
\hline ILUMINAÇÃO & 20 & 6 & 3,6 & 1X20W FLUO & \\
\hline ILUMINAÇÃO & 9 & 6 & 1,62 & 1X9W LED & A \\
\hline ILUMINAÇÃO & 50 & 3 & 4,5 & 1X50W INC & \\
\hline ILUMINAÇÃO & 15 & 6 & 2,7 & 1X15W FLUO & \\
\hline ILUMINAÇÃO & 6,5 & 6 & 1,17 & 1X6,5W LED & $A$ \\
\hline ROTEADOR & 30 & 24 & 21,6 & ROTEADOR NET WIFI & \\
\hline TELEVISAO & 90 & 4 & 10,8 & TELEVISAO & A \\
\hline VIDEOGAME & 175 & 0,5 & 2,625 & $\mathrm{XBOX}$ & \\
\hline VENTILADOR & 100 & 4 & 12 & $\begin{array}{l}\text { VENTILADOR DE } \\
\text { MESA }\end{array}$ & A \\
\hline CLIMATIZADOR & 170 & 8 & 40,8 & $\begin{array}{c}\text { CLIMATIZADOR } \\
\text { PORTATIL } \\
\end{array}$ & A \\
\hline ILUMINAÇÃO & 18 & 0,5 & 0,27 & $\begin{array}{c}\text { LAMPADA TUBULAR } \\
18 \mathrm{~W} \text { LED }\end{array}$ & A \\
\hline ILUMINAÇÃO & 18 & 4 & 2,16 & $\begin{array}{c}\text { LAMPADA TUBULAR } \\
18 \mathrm{~W} \text { LED }\end{array}$ & A \\
\hline ILUMINAÇÃO & 36 & 4 & 4,32 & $\begin{array}{c}\text { LAMPADA TUBULAR } \\
\text { 36W LED }\end{array}$ & A \\
\hline FORNO & 2000 & 0,333 & 19,98 & $\begin{array}{c}\text { FORNO ELÉTRICO } \\
2000 \mathrm{~W} \\
\end{array}$ & \\
\hline FREEZER F1 & 200 & 24 & 144 & $\begin{array}{c}\text { FREEZER ESTOQUE } \\
\text { HORIZONTAL } \\
\text { CONVENCIONAL } \\
\end{array}$ & \\
\hline FREEZER & 115 & 24 & 82,8 & $\begin{array}{c}\text { FREEZER VENDAS } \\
\text { VERTICAL } \\
\text { CONVENCIONAL } \\
\end{array}$ & \\
\hline FREEZER & 130 & 24 & 93,6 & $\begin{array}{c}\text { FREEZER } \\
\text { CONGELAMENTO } \\
\text { VERTICAL } \\
\text { CONVENCIONAL }\end{array}$ & \\
\hline FREEZER & 130 & 24 & 93,6 & $\begin{array}{c}\text { FREEZER } \\
\text { CONGELAMENTO }\end{array}$ & \\
\hline
\end{tabular}




\section{CONWEESSOO CIENCIAESOCIEDADE \\ Inovação, Diversiliaile e Sustentalililitaile}

ANAIS CBCS 2019 | 3 a 5 de outubro de 2019 | Centro Universitário Santo Agostinho - Teresina - P|

\begin{tabular}{|c|c|c|c|c|}
\hline & & & & $\begin{array}{c}\text { VERTICAL } \\
\text { CONVENCIONAL }\end{array}$ \\
\hline GELADEIRA & 231 & 24 & 166,32 & $\begin{array}{c}\text { GELADEIRA } \\
\text { CONVENCIONAL }\end{array}$ \\
\hline $\begin{array}{c}\text { MÁQUINA DE } \\
\text { LAVAR } \\
\text { ROUPAS }\end{array}$ & 310 & 3 & 27,9 & $\begin{array}{c}\text { MÁQUINA DE LAVAR } \\
\text { ROUPAS 10KG }\end{array}$ \\
\hline TOTAL & & & 1176,97 & \\
\hline
\end{tabular}

Fonte: Próprio autor

A partir da análise da atual condição da residência e mediante as necessidades dos membros da casa, foram realizadas algumas propostas de modificações na planta da casa, a fim de torná-la com uma eficiência energética mais expressiva.

A planta a seguir apresenta a sugestão de uma nova planta, baseada na planta atual:

Figura 7: Planta sugerida

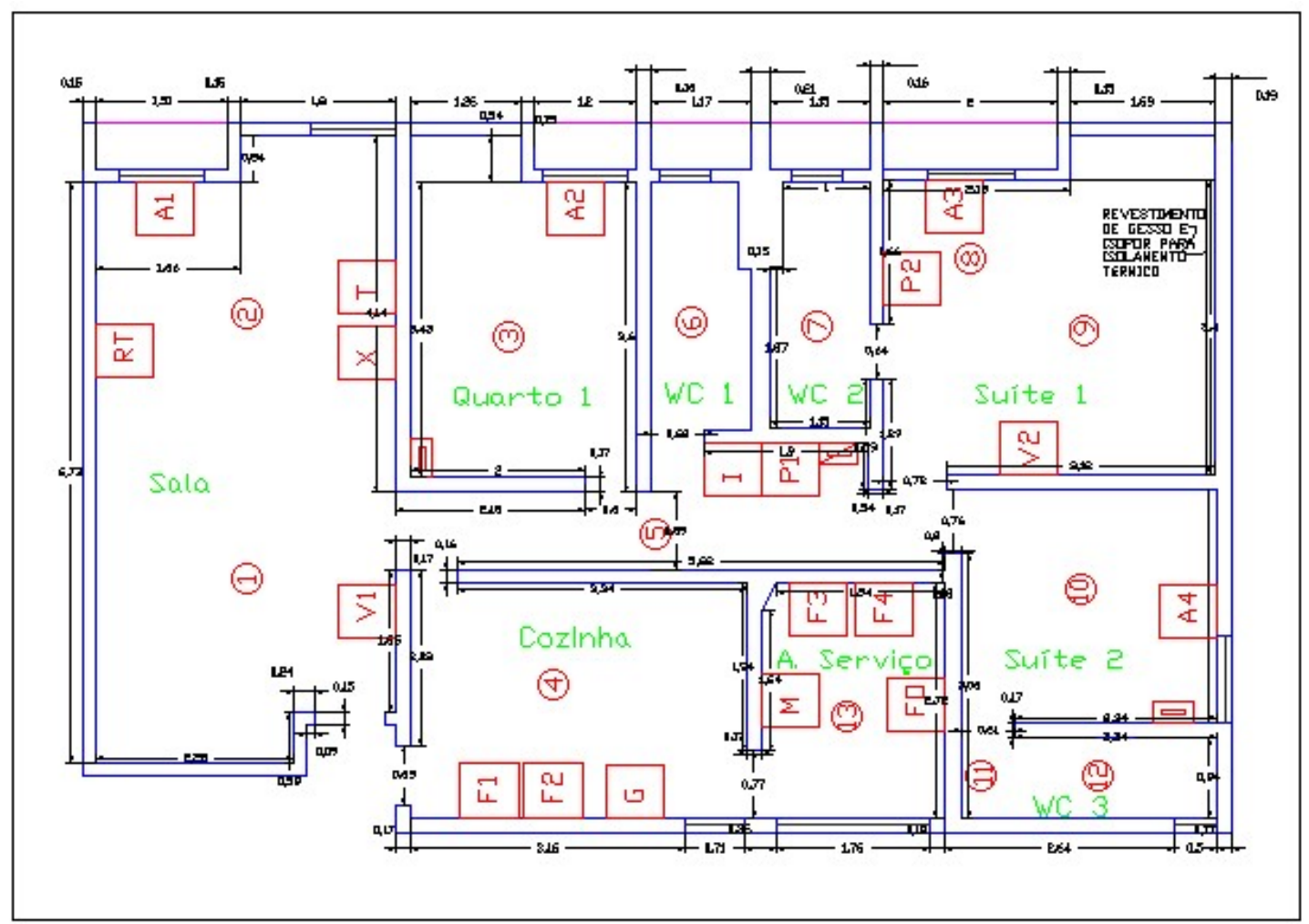

Fonte: Próprio autor 
ANAIS CBCS 2019 | 3 a 5 de outubro de 2019 | Centro Universitário Santo Agostinho - Teresina - P|

Devido à planta em estudo ser de um apartamento em um condomínio, não há a possibilidade de aplicar estas sugestões de alterações, ou são tão burocráticas que se tornam inviáveis. No entanto poderiam ser aplicadas em outros casos que se assemelham e tenham a liberdade de implantar o projeto de reforma.

A partir da análise da planta atual da casa, do levantamento de cargas, das condições físicas e elétricas da residência e com a percepção da sensibilidade dos membros da casa diante a utilização consciente da energia elétrica, foram levantadas medidas de implantação do projeto de eficiência energética, como descrito abaixo:

- Realizar a troca da lâmpada incandescente e fluorescente por lâmpadas de LED, que são inicialmente mais caras, porém são mais econômicas em longo prazo;

- Desligar o roteador no intervalo de 00h00min até as 05 h00min da manhã. Este intervalo é uma média, no caso a sugestão é que durante a noite, quando não se utiliza a internet, que ela seja mantida desligada da tomada, pois mesmo sem uso ainda há consumo de energia quando está no modo stand-by;

- Colocar sensores de LED no corredor e nos banheiros, pois esta é uma maneira de controlar de forma eficaz que essas lâmpadas sejam desligadas quando não há ninguém nos cômodos;

- Em caso de necessidade de trocar o ar condicionado (por exemplo, por defeito), é sugerido que possa escolher um ar condicionado que possua tecnologia inverter;

- No caso de necessidade de uso e de troca do ar condicionado A1, trocar por um equipamento com selo PROCEL A;

- Realizar o desligamento do freezer horizontal (F1) durante a noite, no intervalo de 00h00min até as $05 \mathrm{~h} 00 \mathrm{~min}$ da manhã. Isso deve ser realizado, pois um dos membros já realizou o teste de desligar este freezer durante a noite e este não perdeu a temperatura em que trabalha, uma vez que o mesmo permaneceu fechado toda a noite. Então, é sugerido que esta medida seja realizada algumas vezes durante a semana, pois o consumo do freezer é elevado (144kWh/mês); 
ANAIS CBCS 2019 | 3 a 5 de outubro de 2019 | Centro Universitário Santo Agostinho - Teresina - P

- Retirar os equipamentos de stand-by quando não estiverem em uso: televisão, XBOX, forno elétrico e máquina de lavar. Isso por que em stand-by ainda há consumo de energia elétrica, mesmo que em menor proporção.

Há pouco tempo, a sociedade não possuía um conhecimento das limitações dos recursos naturais e dos efeitos provocados pelo uso exacerbado dos combustíveis fósseis, por exemplo. Atualmente, com as evidências das mudanças climáticas, o aquecimento global, a chuva ácida, a destruição da camada de ozônio e a escassez de energia, a humanidade começa a perceber a necessidade de enfrentar tais problemas. Diante disso, a conservação de energia elétrica torna-se uma opção para a busca de soluções e sistemas mais eficientes, como a utilização de energias renováveis (PUCRS, 2010).

A mudança de hábito é imprescindível para que a população contribua com o consumo eficiente. No que se refere ao uso da energia, a maioria das atitudes estão relacionadas a mudanças de comportamento e, portanto, envolvem uma abordagem que exige significativos esforços educacionais, em curto e longo prazo.

Para a conscientização dos indivíduos que residem na residência em análise, será realizado um momento de esclarecimento acerca da atual situação de consumo da residência e dos pontos que necessitam de mais atenção para atingir a economia almejada. Em seguida, serão repassadas a todos as seguintes sugestões de mudança de hábitos:

Para uso da máquina de lavar roupas:

- Usar a máquina só depois de ter juntado a quantidade de roupa da capacidade máxima do equipamento.

- Limpar com frequência o filtro das lavadoras de roupas.

- Utilizar a quantidade correta de sabão ou detergente para não ter que enxaguar novamente. Para uso do ferro elétrico:

- Esperar acumular uma razoável quantidade de roupas e passá-las de uma só vez. 


\section{CONQGEESSO CIENCIAESOCIEDADE

ANAIS CBCS 2019 | 3 a 5 de outubro de 2019 | Centro Universitário Santo Agostinho - Teresina - PI

- Seguir as instruções de temperatura para cada tipo de tecido, passando primeiramente os tecidos mais grossos, que necessitam temperaturas elevadas, e, após, desligue o ferro e passe os mais leves.

- Evitar ligar o ferro simultaneamente com outros aparelhos elétricos, pois esse eletrodoméstico sobrecarrega a rede de energia elétrica.

Para utilização da televisão:

- Não deixar a TV ligada quando não estiver assistindo.

- Evitar dormir com a TV ligada.

- Dar preferência para aparelhos com timer (função de desligamento automático).

- Durante o período em que se encontra em stand-by, a televisão continua a consumir energia, por isso é necessário retirar este aparelho da tomada quando não estiver em uso.

Para todos os equipamentos:

- Preferir equipamentos com selo Procel (Programa de Conservação de Energia Elétrica): ao comprar lâmpadas ou eletrodomésticos é importante escolher os que possuam o selo Procel, pois garante que o produto esteja entre os mais eficientes do mercado, ou seja, gaste menos energia elétrica do que produtos semelhantes que não tenham o selo. A etiqueta informa sobre a eficiência dos equipamentos, por exemplo, um aparelho classificado como " $A$ " é considerado mais eficiente e econômico do que o mesmo aparelho classificado como " $G$ ", que é o menos viável economicamente.

Além de essas informações serem repassadas de maneira a conscientizar os membros da casa, através de uma conversa de estímulo e conscientização e levando conhecimento da importância do projeto, será realizada apresentação das metas e dos resultados esperados.

Ademais, serão confeccionados materiais de divulgações, como cartazes, e fixação deles em pontos estratégicos da residência, principalmente próximos aos interruptores de iluminação e de disjuntores de ar condicionado.

\section{RESULTADOS E DISCUSSÃO}




\section{CONGRESSO BRASLEERO

ANAIS CBCS 2019 | 3 a 5 de outubro de 2019 | Centro Universitário Santo Agostinho - Teresina - PI

A partir das necessidades de troca de alguns dos atuais equipamentos utilizados na residência e na inclusão de outros, foi realizado o levantamento do material que será utilizado para a implantação do projeto e seu respectivo custo, como mostra a Tabela 2:

Tabela 2. Material e custo para implantação do projeto

\begin{tabular}{|c|c|c|c|c|c|c|}
\hline ITEM & DISCRIÇÃO & QTE & $\begin{array}{c}\text { VALOR } \\
\text { UN }\end{array}$ & $\begin{array}{c}\text { TOTAL } \\
\text { R } \$\end{array}$ & $\begin{array}{c}\text { LOJA DA } \\
\text { PESQUISA }\end{array}$ & ENDEREÇO \\
\hline 1 & LÂMPADA LED 8W & 4 & 23,20 & 92,8 & Americanas & $\begin{array}{c}\text { Av. Dom Severino } \\
1599\end{array}$ \\
\hline 2 & $\begin{array}{c}\text { SENSOR DE } \\
\text { PRESENÇA }\end{array}$ & 4 & 15 & 60 & Piauino & $\begin{array}{c}\text { R. Gen. Adelmar } \\
\text { Rocha, 2525 - } \\
\text { Ininga, }\end{array}$ \\
\hline 3 & $\begin{array}{c}\text { FILTRO DE LINHA 5T } \\
4\end{array}$ & 2 & 17,9 & 35,8 & Americanas & $\begin{array}{c}\text { Av. Dom Severino } \\
1599\end{array}$ \\
\hline 5 & $\begin{array}{c}\text { 12000 BTU INVERTER } \\
\text { LG }\end{array}$ & 1 & 1799 & 1799 & Americanas & $\begin{array}{c}\text { Av. Dom Severino } \\
1599\end{array}$ \\
\hline $\begin{array}{c}\text { REISOLAMENTO } \\
\left.\text { ISOPOR(M }{ }^{2}\right)\end{array}$ & 18,5 & 50 & 925 & $\begin{array}{c}\text { Ranieri } \\
\text { Gesso }\end{array}$ & $\begin{array}{c}\text { São Sebastião Q E C } \\
5\end{array}$ \\
\hline \multicolumn{2}{|c|}{ TOTAL } \\
\hline
\end{tabular}

Fonte: Próprio autor

Os equipamentos que serão trocados são: um ar condicionado de 12000 BTU, por um de tecnologia inverter, duas lâmpadas fluorescentes de 20W, uma lâmpada fluorescente de $15 \mathrm{~W}$ e uma lâmpada incandescente de 50W, por quatro lâmpadas de LED de $8 \mathrm{~W}$ cada. Além disso, será comprado um filtro de linha $5 T$ para conectar a televisão e o videogame, podendo estes serem desligados quando não estiverem sendo utilizados, a fim de evitar o stand-by, que consome energia elétrica.

Também será realizado o isolamento térmico da parede que abrange a suíte 1 e 2 , havendo então a necessidade da compra de gesso. Essa necessidade foi percebida devido ao comportamento do sol ao longo do dia em relação à residência, pois durante parte da tarde e no pôr do sol esta parede recebe irradiação solar bastante intensa, aquecendo esses ambientes. Outra mudança será 


\section{cONQ⿻口卄:ESOC CIENCIASSOCIEDADE

ANAIS CBCS 2019 | 3 a 5 de outubro de 2019 | Centro Universitário Santo Agostinho - Teresina - PI

a programação de desligamento do freezer (F1) e do roteador por um período de 5 horas durante a noite, como explicado anteriormente.

Anteriormente às modificações, o total do consumo, calculado a partir do levantamento de cargas foi de $1176,97 \mathrm{Wh} / \mathrm{mês}$. Após as mudanças implementadas pelo projeto o consumo passou a ser de aproximadamente 1142,34KWh/mês:

Diante disso, calculando a divisão entre o consumo verificado após as sugestões e o consumo calculado antes do projeto, tem-se a porcentagem de consumo futuro em relação ao atual:

$$
\frac{1142,34}{1176,97}=0,97057
$$

Subtraindo esse valor de 100\% tem-se aproximadamente a eficiência alcançada no consumo de energia elétrica:

$$
1-0,97057=2,94 \%
$$

Portanto, considerando a taxa de $0,868147 / \mathrm{kWh}$, o mesmo contido nas faturas, tem-se que:

Fatura atual: $\mathrm{R} \$ 1021,78$

Fatura após projeto: $\mathrm{R} \$ 991,71$

Uma vez que os custos de implantação do projeto foram de $\mathrm{R} \$ 2912,6$, o retorno financeiro será alcançado em aproximadamente 8 anos.

\section{CONSIDERAÇÕES FINAIS/CONCLUSÕES}

Diante do exposto, foi possível verificar que a residência a qual o projeto foi sugerido é considerada um caso atípico, pois esta já apresentava, em sua maioria, equipamentos bastante eficientes, com selo Procel A, com tecnologia inverter, utilizando também equipamentos específicos (vários freezers), além de se tratar de um condomínio, e, por tudo isso, apresentou poucas possibilidades de implantação de melhorias no quesito eficiência energética.

Diante do cálculo da fatura já com a eficiência e utilizando a tarifa de energia elétrica atual, foi possível observar que o payback terá uma duração de aproximadamente 8 anos, o que apresenta um resultado razoável, uma vez que a residência já apresenta muitas características eficientes. 


\section{conghisSO CIENCIAESOCIEDADE

ANAIS CBCS 2019 | 3 a 5 de outubro de 2019 | Centro Universitário Santo Agostinho - Teresina - PI

Com o desenvolvimento do projeto, foi possível concluir que apesar de muito eficiente, ainda há melhorias que podem ser alcançadas na economia da conta de energia, porém, como ela apresenta tais características já mencionadas, a eficiência alcançada não foi tão significativa, podendo ser maior em um empreendimento com menos equipamentos eficiente, por exemplo.

\section{REFERÊNCIAS}

Atlas de energia elétrica do Brasil / Agência Nacional de Energia Elétrica. 3. ed. - Brasília: Aneel, 2008.

Economia e a Administração: mercado e poder. Disponível em: $<$ http://www.econoteen.fea.usp.br/o-que-e-economia>Acesso em: 23 maio 2019.

Eficiência

Energética.Disponível

em:

$<$ http://www.inee.org.br/eficiencia_o_que_eh.asp?Cat=eficiencia $\geq$ Acesso em: 28 maio 2019.

Manual de tarifação de energia / A. ELETROBRAS, Procel e Edifica Procel. - 2011.

MARQUES, M. C. S., HADDAD, J., GUARDIA, E. C., et al., Eficiência energética: teoria e prática, Itajubá, MG, Brasil: FUPAI, 2007

PÓVOA, M. C. B. L. FATORES DE INFLUÊNCIA NA EFICIÊNCIA ENERGÉTICA. Universidade federal do Rio de Janeiro. Rio de Janeiro, 2014. Disponível em: $<$ http://monografias.poli.ufrj.br/monografias/monopoli10010700.pdf $>$. Acesso em: 21 maio 2019. 